\title{
9 Der alte Patient in der Notaufnahme - Entscheidungen am Lebensende
}

\author{
Stefan Trzeczak
}

\subsection{Einführung}

Über 50\% der Patienten in der Akutmedizin oder in der Notaufnahme sind betagte, multimorbide Patienten (Hien et al. 2013, S. V). Die mittlere Lebenserwartung für Frauen und Männer in Europa und damit der Anteil der über 65-Jährigen an der Gesamtpopulation sind in den letzten Jahrzehnten deutlich angestiegen (Schreiber 2014). Ursächlich für den demografischen Wandel sind die anhaltend niedrige Geburtenhäufigkeit sowie der kontinuierliche Anstieg der Lebenserwartung. Zuwanderungsprozesse haben den Alterungsprozess der Cesellschaft „nur abbremsen, aber nicht aufhalten“ können (Bäcker et al. 2010).

Es wird ein „dreifaches Altern der Gesellschaft“ unterschieden: Die Zunahme der absoluten Zahl älterer Menschen, der wachsende Anteil älterer Menschen an der Gesamtbevölkerung (durch die „geburtenschwachen Jahrgänge“) sowie der starke Anstieg der sehr alten Menschen im Alter von 80 Jahren und mehr (durch die Zunahme der Lebenserwartung).

Dabei hat sich die Zahl der 6o Jahre alten und älteren Menschen in Deutschland von 16,3 Mio. 1990 über 18,4 Mio. im Jahr 2000 bis 20,568 Mio. im Jahr 2006 entwickelt, der Anteil der 6o-jährigen und älteren Menschen stieg von 20,4\% 1990 über 22,4\% 2000 bis 25,0\% 2006, die Anzahl der 8o-jährigen und älteren Menschen stieg von 3,011 Mio. 1990 auf 3,77 Mio. 2006, das bedeutet einen Bevölkerungsanteil von 3,8\% 1990 und 4,5\% 2006 (s. Tab. 12). 
Tab. 12 Entwicklung von Anzahl und Anteil der über 60-ährigen und über 80-Jährigen an der Gesamtbevölkerung in Deutschland (nach Bäcker et al. 2010)

\begin{tabular}{|l|cccc|}
\hline Jahr & Anzahl $>60 \mathrm{~J}$. & Bev-Anteil $>60$ J. & Anzahl $>80$ J. & Bev-Anteil > $80 \mathrm{~J}$. \\
\hline 1990 & 16,3 Mio. & $20,4 \%$ & 3,011 Mio. & $3,8 \%$ \\
\hline 2000 & 18,4 Mio. & $22,4 \%$ & - & - \\
\hline 2006 & 20,568 Mio. & $25,0 \%$ & 3,77 Mio. & $4,5 \%$ \\
\hline
\end{tabular}

Prognostisch müssen wir mit einem Rückgang der Gesamtbevölkerung in Deutschland (von 82,3 Mio. 2006 auf 79,8 Mio. 2030 und 74 Mio. 2050) sowie mit einem steigenden Anteil der über 6o-Jährigen auf 35,8\% 2030 und 38,8\% 2050 sowie der über 8o-Jährigen von 3,9\% 2006 auf 10,7\% 2050 rechnen (s. Tab. 13, Bäcker et al. 2010).

Tab. 13 Prognose der Entwicklung von Gesamtbevölkerung und Anteil der über 60-ährigen und über 80-ährigen an der Gesamtbevölkerung in Deutschland (nach Bäcker et al. 2010)

\begin{tabular}{|c|ccc|}
\hline Jahr & Gesamtbevölkerung & Bev-Anteil > 60 J. & Bev-Anteil > 80 J. \\
\hline 2006 & 82,3 Mio. & $25,0 \%$ & $3,9 \%$ \\
\hline 2030 & 79,8 Mio. & $35,8 \%$ & - \\
\hline 2050 & 74 Mio. & $38,8 \%$ & $10,7 \%$ \\
\hline
\end{tabular}

Dabei zeigt sich auch ein Strukturwandel des Alters: Die Lebensphase, in der jemand als „alt“ bzw. „älter“ angesprochen wird, verlängert sich, da sie meist die Zeit vom Berufsausstieg bis zum Ableben bezeichnet. Dies führt innerhalb dieser Lebensphase noch zu weiteren Unterscheidungen, z.B. zwischen „jungen Alten“, „mittleren Alten“ und „Hochaltrigen“. Während die ,jungen Alten“ und die „mittleren Alten“ häufig noch sehr aktiv sind (was man bei der Planung einer Behandlung berücksichtigen muss), kommen bei den „Hochaltrigen“ häufig Multimorbidität und geriatrische Syndrome zum Tragen. Weiterhin ist auffällig, dass das Bild der älteren Bevölkerung heutzutage hauptsächlich von Frauen geprägt wird - bedingt durch die höhere Lebenserwartung der Frauen und die höhere Mortalität der Männer während des Zweiten Weltkrieges. Ein zusätzliches Merkmal ist, dass die Menschen in höherem Alter vermehrt allein leben. Und schließlich ist ein besonderes Merkmal die Hochaltrigkeit, d.h. ein Alter von 80 Jahren und älter. Das heißt aber nicht, dass wir es bei steigender Zahl älterer Menschen automatisch mit mehr kranken Menschen zu tun haben: Untersuchungen in Österreich, die sich aber problemlos auf andere westliche mitteleuropäische Länder übertragen lassen, haben gezeigt, dass die Lebenserwartung bei guter Gesundheit überwiegt (Klotz 2013). Der Terminus „gute Gesundheit“ ist aber ein bei Befragungen subjektiv erhobener Parameter - der Autor führt weiter aus, dass die Lebenserwartung ohne merkbare funktionale Beeinträchtigung zwar 2003 bis 2011 bei Männern um 2,9 Jahre und bei Frauen um 1,7 Jahre stieg, aber dass die Lebenserwartung ohne chronische Erkrankung im gleichen Zeitraum bei Männern um o, 8 Jahre und bei Frauen um 3 Jahre zurückging. Das heißt, dass die Menschen zwar älter werden, aber im Laufes des Lebens häufiger an chronischen Erkrankungen leiden (die sie aber offensichtlich wenig im Alltag beeinträchtigen - trotzdem haben wir dies bei unse- 
rem Kontakt mit diesen Menschen, wenn sie uns als Patienten aufsuchen, zu berücksichtigen). US-amerikanische Untersuchungen haben gezeigt, dass „durch die besseren medizinischen Behandlungsmethoden zwar die krankheitsbezogene Mortalität sinkt und damit auch die Lebenserwartung steigt, dies aber mit einer zunehmenden Zahl an multimorbiden Menschen einhergeht“ (Köller 2014).

Ältere Menschen suchen zunehmend häufiger die Notaufnahmen auf, auch ohne eine klassische „Notfallindikation“ (Lowthian et al. 2013). Ein Grund dafür ist die Schwierigkeit, beim Hausarzt oder Facharzt zeitnah einen Termin zu bekommen, verbunden mit Erwartungen an schnellere und kompetentere Hilfe. Eine andere Studie zeigte jedoch, dass $74,2 \%$ aller Patienten über 65 Jahre in einer Notaufnahme (Halifax) als bedroht oder dringend eingestuft wurden und teilweise umfangreiche bildgebende und Labordiagnostik durchliefen, was zu einer längeren Verweildauer dieser Patienten in der Notaufnahme führte, verbunden mit der Gefahr unerwünschter Ereignisse während des Notaufnahmeaufenthaltes (Latham u. Ackroyd-Stolarz 2014).

Das alles projiziert sich vor den Hintergrund einer immer leistungsfähigeren Hightech-Medizin einerseits und zunehmend praktizierter Patientenautonomie in Form von Patientenverfügungen, Vorsorgevollmachten und „Advanced-Care-Planning“ andererseits.

Noch nie in der Geschichte der Medizin waren die Möglichkeiten, „alles zu tun“, so groß und gleichzeitig die Mittel des Patienten, „alles abzulehnen“, so juristisch abgesichert wie heute.

In der Diskussion darüber finden sich Äußerungen von Kollegen in einer Spannbreite von: „Im Einzelfall müssen wir uns fragen, ob nicht ein plötzlicher Tod durch einen schmerzlosen Herzstillstand von dem Betroffenen auch als eine Gnade empfunden werden kann?“ (Kettler 1997) bis „Wer wird schon gerne 9o? Die 89-Jährigen!“ (Grönemeyer 2003).

In diese Fülle von Fakten und sich widersprechenden Möglichkeiten und Meinungen etwas Licht $\mathrm{zu}$ bringen und dabei möglichst noch eine Verbesserung der Betreuung unserer immer älter werden Patienten zu erreichen, soll Ziel der folgenden Seiten sein. Dies geschieht nicht ganz uneigennützig - auch wir werden früher oder später zu der Patientenklientel gehören, um die es hier gehen soll.

\subsection{Der alte Patient}

„Das Altern gehört wie Geburt und Tod zu den Urphänomenen des Lebens. Es stellt einen Vorgang dar, der mit der Geburt beginnt und unter ständigen Wandlungen seiner körperlichen Substanz mit dem physiologischen Tode endet ... Nascentes morimur ${ }^{7}$, ein Ausdruck, der von Seneca stammen soll aus Brief 4, s g: ex quo natus est, duceris ${ }^{8}$, d.h. zur Hinrichtung abgeführt.“ (Bürger 1960)

7 "Schon wenn wir geboren werden, sterben wir"

8 „von dort, wo ich geboren wurde, abgeführt“ 
Dieses Altern verläuft aber nicht bei allen Menschen gleich schnell, sondern ist u.a. abhängig von Faktoren wie ,alles, was dem Menschen ... an seelischem, geistigem und körperlichem Gewinn zuströmt, und Einwirkungen, die ihn durch Nahrung, Klima, Übung und Beruf beeinflussen“, also „die Biographie der Person“ (Bürger 1960). Es ist also ein „schicksalsmäßig ablaufender irreversibler Vorgang“ (ebd.).

Wegen dieser vielfältigen interindividuellen Unterschiede der Alterungsvorgänge wird in der Literatur und der klinischen Praxis zwischen kalendarischem und biologischem Alter unterschieden (Tschugg 2012). Wenn wir uns in diesem Kapitel dem älteren Patienten zuwenden, ist damit der Patient nach der gemeinsamen Definition der ÖGGC und der DGGC gemeint:

\section{Definition geriatrischer Patient:}

„Der geriatrische Patient ist ein biologisch älterer Patient, der durch altersbedingte Funktionseinschränkungen bei Erkrankungen akut gefährdet ist, zur Multimorbidität neigt und bei dem ein ganz besonderer Handlungsbedarf in rehabilitativer, somatopsychischer und psychosozialer Hinsicht besteht" (aus: Pinter et al. 2013).

Dabei ist die geriatrietypische Multimorbidität vorrangig vor dem kalendarischen Alter zu sehen (Heppner et al. 2014).

Der menschliche Körper macht mit zunehmendem Alter typische somatische Veränderungen durch (Schippinger et al. 2013): Die zerebrale Katecholaminsynthese nimmt $\mathrm{ab}$, dies begünstigt die Entstehung von Depressionen. Eine Abnahme der zerebralen Dopaminsynthese kann zu M. Parkinson und eine neuronale Atrophie mit hauptsächlich zahlenmäßiger Abnahme der Dendriten zu kognitiven Defiziten bis hin zur Demenz führen. Das renovaskuläre Gefäßnetz rarefiziert, dies führt zur Abnahme des renalen Plasmaflusses und damit der glomerulären Filtrationsrate und der Fähigkeit zur Harnkonzentration bzw. -dilution. Parenchymatrophie führt außerdem zur Abnahme der 1-Hydroxylaseaktivität. Blutdruckbelastung führt einerseits zur Vorhofvergrößerung des Herzens und begünstigt die Entstehung von Vorhofflimmern, andererseits zur Myokardverdickung mit Abnahme der Kontraktilität und der Herzfrequenzvariabilität mit der Folge einer geringeren kardialen Reserve unter Belastung. Abnahme der Gefäßwandelastizität hat die Neigung zu systolischer arterieller Hypertonie zur Folge. Die Elastizität (Compliance) der Lunge und die Kraft der Atemmuskulatur nehmen ab, ebenso die mukoziliäre Clearance und der Hustenreflex, dies bedingt eine Abnahme des pulmonalen Gasaustausches und Neigung zu bronchopulmonalen Infekten. Veränderungen der Haut führen u.a. zu gestörter Thermoregulation, eine Abnahme der Knochenmarksaktivität zu Störungen der Hämatopoese, Abnahme der Knochendichte zu gehäuften Frakturen sowie in Verbindung mit Sarkopenie zu zunehmender Sturzhäufigkeit, außerdem kommt es zu Abnahme der T-Zell-Aktivität mit Auswirkungen auf das Immunsystem und zu steigendem Risiko für Autoantikörperbildung. Dies sollen nur einige Beispiele sein, die die biologischen Alterungsvorgänge illustrieren. Diese Veränderungen zeigen sich am Patienten in Form von Multimorbidität und „Frailty“.

\section{Definition von „Multimorbidität“:}

„... wird definiert als jegliche Kombination einer chronischen Erkrankung mit zumindest einer weiteren Erkrankung (akut oder chronisch), einem biopsychosozialen Faktor (assoziiert oder nicht) oder einem somatischen Risikofaktor" (Wutti 2014). 
Als „Frailty“ wird eine Art „geriatrietypische Gebrechlichkeit“ beim älteren Patienten bezeichnet:

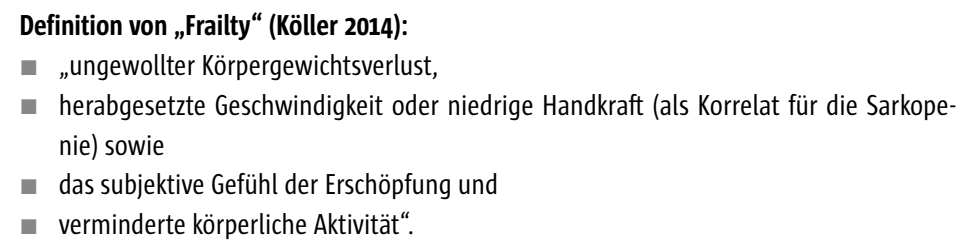

Dieser Definition zufolge sind im deutschsprachigen Raum lt. Literaturangaben ca. 10\% der über 65-Jährigen als „frail“ und weitere $40-45 \%$ als „pre-frail“ zu betrachten (Köller 2014).

Multimorbidität zieht Polypharmazie nach sich, wobei in der Pharmakotherapie beim älteren Patienten einmal mehr die alterstypischen somatischen Veränderungen zu beachten sind: Die enterale Resorption und die Distribution über den Blutstrom sind eingeschränkt, was möglicherweise zu einer anderen als der erwarteten Wirksamkeit führt. Gleichzeitig sind aber der first-pass-Metabolismus der Leber, der enterohepatische Kreislauf und sowohl die hepatische als auch die renale Elimination eingeschränkt, was häufig eine Dosisanpassung erforderlich macht bzw. die Benutzung bestimmter Medikamente zumindest kritisch erscheinen lässt. Doch auch die Pharmakodynamik, also die Interaktion eines Medikaments mit seiner Zielstruktur bzw. seinem Rezeptor, ist im Alter Veränderungen unterworfen (Schippinger et al. 2013). Wegen dieser skizzierten Änderungen der Pharmakaverträglichkeit entsteht folgendes Dilemma:

„Die moderne Medizin unterhält einen unüberschaubaren Markt von Pharmazeutika, die für den Einzelfall gründlich getestet und wirksam sind, für den alten, multimorbiden Patienten im Rahmen der Polypharmakotherapie hingegen eine Gefahr bedeuten können. Es besteht das Dilemma, dass die Altersgruppen, die am häufigsten Ärzte aufsuchen und die die meisten Medikamente verordnet bekommen, in der pharmakologischen Forschung und in der Struktur des medizinischen Alltags am schlechtesten berücksichtigt sind. Mit ihrem biologisch alten Körper gehorchen die Alten mitunter eigenen Maßstäben und unterstehen als Multimorbide einem besonders hohen Risiko unbekannter pharmazeutischer Interaktionen." (Krug 2009)

Wir haben es also insgesamt mit einer Patientengruppe zu tun, die sich einerseits durch Gebrechlichkeit, Multimorbidität und komplizierende biopsychosoziale Faktoren auszeichnet und zum anderen eben durch diese Multimorbidität sowie durch Veränderungen des Stoffwechsels, der Erfolgsorgane und der zerebralen Funktion (Compliance!) eine besondere Herausforderung für therapeutisches Tätigwerden darstellen.

\subsection{Der alte Patient in der Notaufnahme}

Notaufnahmen sind auf Effizienz, Schnelligkeit und Erlös ausgerichtete Einrichtungen, in denen möglichst vielen Patienten in möglichst kurzer Zeit die bestmöglich adäquate Hilfe zu Teil werden soll, abgestuft nach Akuität ihrer Beschwerden bzw. 
Dringlichkeit ihrer Behandlungsbedürftigkeit (Cernic et al. 2013). Wie tritt uns jetzt ein „älterer Patient“, dessen Besonderheiten im vorigen Abschnitt kurz charakterisiert wurden, in so einem auf Schnelligkeit und Effizienz getrimmten Betrieb gegenüber?

„Ein alter Patient ist alt.“ (Krug 2009)

Dies hat zur Folge, dass er dem mit ihm in Kontakt tretenden medizinischen Personal mehr Zeit abfordert. Er ist langsamer in seinen Verrichtungen (z.B. Ausziehen, Hinlegen ...), im Sprechen, im Denken und fordert letztendlich einen höheren Zeitbedarf bei der Untersuchung. Hinzu kommt ein meist entspannter Zeitrahmen eines nicht mehr im Berufsleben stehenden Patienten und nicht zuletzt die Einsamkeit vieler älterer Menschen, die den Arztkontakt gleichzeitig als einen wertvollen, weil seltenen sozialen Kontakt wahrnehmen und beim Arzt/bei der Pflegeperson bzw. dem Rettungsdienst nicht nur Rat und Hilfe, sondern auch eine zwischenmenschliche Kommunikation suchen (Krug 2009).

„Unspezifische Beschwerden führen erst nach langem Nachfragen und ausgiebiger Untersuchung zur richtigen Diagnose, und selbst komplexe Erkrankungssituationen präsentieren sich durch die verminderte oder atypisch ausgeprägte Symptomatik weniger eindrucksvoll, sind aber dennoch vital bedrohlich." (Heppner et al. 2014)

Ein Fünftel aller älteren Patienten stellt sich mit unspezifischen Symptomen in einer Notfallambulanz vor (Köller 2014). Dies führt zu einer Untertriagierung mit falscher Einschätzung der Dringlichkeit von Untersuchungen und den fatalen Folgen. So kann z.B. Fieber als Kardinalsymptom fehlen (ebd.). Viele somatische Erkrankungen zeigen sich als Vigilanzminderung/Somnolenz und werden als „TIA“ fehlgedeutet bzw. imponieren als hyperdynames Delir mit Unruhe, Desorientierung und Agitation, was zu einer Fehleinweisung in die Gerontopsychiatrie führt.

„Eine kanadische Untersuchung an über 2.700 Patienten in einer Notaufnahme, die älter als 65 Jahre waren, hat gezeigt, dass die Dringlichkeit von Untersuchungen bei $26 \%$ der Patienten falsch eingeschätzt wurde. Ursache dafür waren oft die fehlende Erhebung von Vitalparametern, das Übersehen neurologischer Symptome und die atypische klinische Präsentation der Beschwerden“"(Köller 2014).

Typische Fehler in der Anamneseerhebung beim älteren Patienten (nach Cernic et al. 2013):

- mangelhafte Fremd- und Medikamentenanamnese

- mangelhafte Differenzialdiagnose

- Nichterkennen einer Depression

- Nichterhebung eines Sturzrisikos

- Nichterkennen von Infekten wegen fehlenden Fiebers

- Verkennen von Myokardinfarkten bei atypischen Symptomen

- Nichtberücksichtigung des Patientenwunsches nach Therapiebegrenzung

Die häufigsten Symptomkomplexe von älteren Patienten in einer Notaufnahme sind nach Köller (2014) Erkrankungen des Herz-Kreislauf-Systems (30-35\%), Stoffwechselstörungen, besonders Diabetes mellitus (10-15\%), muskuloskelettale Beschwerden (10\%) sowie neurologische und psychiatrische Erkrankungen (10\%). Dabei führt die 
Multimorbidität dieser Patienten dazu, dass diese häufig innerhalb kurzer Zeit erneut in einer Notaufnahme vorstellig werden: „27\% der älteren Patienten, welche von einer Notfallaufnahme entlassen werden, stellen sich innerhalb von 3 Monaten erneut an einer solchen Einrichtung vor, werden stationär aufgenommen oder versterben“ (Köller 2014). Ein valides und in einer Notaufnahme gut handhabbares Instrument, das Risiko eines älteren Patienten für Multimorbidität einzuschätzen und damit auch Entscheidungen über die Notwendigkeit einer stationären Aufnahme zu treffen, ist das sogenannte ISAR-Screening (Köller 2014, s. Tab. 14).

Tab. 14 Indikatoren für Multimorbidität nach ISAR („Identification Seniors at Risk“) (nach Köller 2014)

\begin{tabular}{|l|c|}
\hline Indikatoren & Auswertung \\
\hline Vor Aufnahme zu Hause hilfsbedürftig? & $\mathrm{la}=1$ \\
\hline Durch aktuelle Erkrankung hilfsbedürftig? & $\mathrm{la}=1$ \\
\hline Hospitalisierung (>1 d) in den letzten 6 Monaten? & $\mathrm{la}=1$ \\
\hline Sehen Sie gut? & $\mathrm{Nein}=1$ \\
\hline Haben Sie Gedächtnisprobleme? & $\mathrm{la}=1$ \\
\hline Einnahme von $>3$ Medikamenten am Tag? & $\mathrm{la}=1$ \\
\hline
\end{tabular}

Wenn ISAR > 2 ist, liegen bei $61 \%$ der Patienten > 1 behandlungsbedürftige Erkrankungen vor gegenüber $18 \%$ bei ISAR $0 / 1$.

Dazu muss angemerkt werden, dass Patienten mit einem ISAR > 2 oder $=2$ ein doppelt bis dreifach erhöhtes Risiko für eine Rehospitalisierung aufweisen, deshalb reduziert eine frühe Identifikation dieses Risikos einen späteren Funktionsverlust durch Hospitalisierung (Köller 2014).

\section{Exkurs 1: „Psychose“ oder „Delir“?}

Die neurologische Dysfunktion eines sogenannten Delirs präsentiert sich bei 15-40\% der älteren Patienten bereits bei Aufnahme in der Notfallambulanz, wird jedoch nur zu 50\% der Fälle erkannt (Wutti 2014). Köller (2014) beziffert 20\% Auftreten von akuten Bewusstseinsstörungen bei älteren Patienten in einer Notaufnahme, von denen etwa nur ein Drittel richtig erkannt wird. Aus eigener Erfahrung kann berichtet werden, dass diese Patienten häufig als „Psychose“ eingewiesen werden und es meist nur dem stoischen Insistieren der leidgeprüften Gerontopsychiater zu verdanken ist, dass sie einer somatischen Diagnostik und letztendlich kausalen Therapie zugeführt werden. Es werden drei Formen des Delirs unterschieden: das hyperaktive, das hypoaktive und Mischformen.

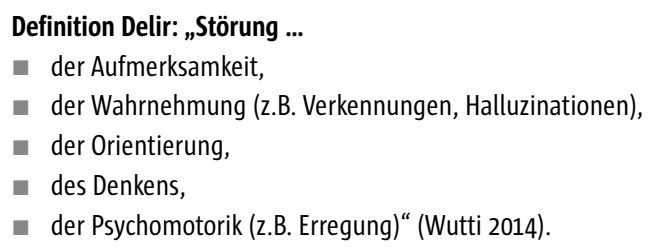


Andere Autoren legen außerdem Wert auf das Plötzliche und Fluktuierende dieser Erscheinungen (Heppner et al. 2014). Einig ist man sich darüber, dass das Delir beim älteren Menschen häufig das erste und einzige Anzeichen einer ernsthaften, mitunter sogar lebensbedrohlichen Erkrankung sein kann (Heppner et al. 2014; Wutti 2014; Köller 2014).

\section{Das Delir ist beim älteren Patienten häufig das erste und einzige Anzeichen einer ernsthaften oder sogar lebensbedrohlichen Erkrankung.}

Die Ursachen eines Delirs können akute Erkrankungen oder medikamentöse Nebenwirkungen sein. Die eigene Erfahrung zeigt, dass häufig Exsikkose, Elektrolytverschiebungen, Blutzuckerentgleisungen, Infekte (Harnwegsinfekt noch häufiger als Pneumonie oder Gastroenteritis), Neuroleptika, Sedativa oder Morphin die Ursache sind (der „Klassiker“: das bei der körperlichen Untersuchung oft übersehene und im Medikamentenplan noch nicht verzeichnete, weil gerade erst neu verordnete „Schmerzpflaster“!)

\section{Exkurs 2: Vigilanzminderung - „IIA“ oder „Sepsis“?}

Häufig werden ältere Patienten mit „Vigilanzminderung“, „Wesensveränderung“ und ähnlichen Symptomen vom Hausarzt, vom Rettungsdienst und vom Notarzt als „TIA“ oder „Apoplex“ eingewiesen und primär dem Neurologen vorgestellt. Eine fokale Neurologie lassen diese Patienten häufig vermissen. Eine häufige Differenzialdiagnose dieser Zustände ist die septische Enzephalopathie. Ursächlich werden hier verschiedene Faktoren, u.a. eine durch die Sepsis bedingte Änderung der Hämodynamik angenommen (Terborg 2012).

„An eine septische Enzephalopathie muss bei Patienten gedacht werden, bei denen eine Enzephalopathie auftritt und eine Sepsis im Raum steht." (Terborg 2012)

An eine Sepsis ist u.a. zu denken, wenn SIRS-Kriterien vorliegen (s. Tab. 15).

\section{Tab. 15 ACCP-SCCM-Konsensuskriterien für SIRS, Sepsis und Organversagen (nach Terborg 2012)}

\begin{tabular}{|c|c|}
\hline Schweregrad & Symptome \\
\hline $\begin{array}{l}\text { SIRS (Systemic Inflammatory } \\
\text { Response Syndrome) }\end{array}$ & $\begin{array}{l}\text { Inflammatorische Reaktion auf eine Vielzahl pathogener Stimuli, wenn } \\
>2 \text { Kriterien positiv: } \\
\text { " Temperatur }>38 \text { oder }<36^{\circ} \mathrm{C} \\
\text { Herzfrequenz }>90 / \mathrm{min} \\
\text { " Atemfrequenz }>20 \mathrm{~min} \text { oder } \mathrm{pCO}_{2}<32 \mathrm{mmHg} \\
\text { Leukos }>12 \text { oder }<4 \mathrm{~g} / \mathrm{l} \text { oder }>10 \% \text { Stabkernige }\end{array}$ \\
\hline Sepsis & SIRS + Infektnachweis \\
\hline Schwere Sepsis & $\begin{array}{l}\text { Sepsis mit Organdysfunktion, Perfusionsstörung oder Hypotension } \\
\text { (< } 90 \mathrm{mmH} \text { m systolisch oder Abfall um } 40 \mathrm{mmHg} \text { oder mehr vom } \\
\text { systolischen Ausgangsblutdruck) }\end{array}$ \\
\hline Septischer Schock & Schwere Sepsis mit Hypotonie trotz adäquatem Flüssigkeitsersatz \\
\hline
\end{tabular}


In unserer Notaufnahme wurde ein einfacher Algorithmus implementiert, um bei neurologischen bzw. psychischen Störungen extrazerebrale organische Ursachen zu detektieren und abzugrenzen:

- Anamnese, wenn nötig Fremdanamnese, wichtig: Medikamentenanamnese!

- körperliche Untersuchung

- Temperaturmessung (Fieber? Untertemperatur?)

- Monitoring (EKG-Überwachung, Blutdruck, Pulsoxymetrie)

- SBH, in der Notaufnahme als vor-Ort-(,,POCT“)-Leistung verfügbar ( $p H$ ? Hb/Hk? $\mathrm{pO}_{2}$ ? $\mathrm{pCO}_{2}$ ? BZ? Na? K? Lactat? BE? Bicarbonat?)

- ggf. weitere laborchemische Untersuchungen wie z.B. Leberwerte, Kreatinin (Urämie? Leberkoma?) oderMedikamentenscreening (Digitoxin-, Opiatspiegel?)

- ggf. Hinzuziehung des Neurologen und nach neurologischer Untersuchung ggf. Kopf-CT

Mit dieser kurzen Aufzählung an schnell durchgeführten Untersuchungen lassen sich bereits eine Menge extrakranieller Ursachen für Vigilanzminderung herausfinden und somit die Untersuchungszeit für die älteren Patienten verkürzen. Wenn eine Hypoglykämie bzw. ein hyperosmolares Koma beim Diabetes als Ursache für die Vigilanzminderung herausgefunden wird, bleibt dem Patienten möglicherweise längere Wartezeit auf den Neurologen bzw. ein Kopf-CT erspart, und die Therapie kann zeitnah einsetzen.

Aus Exkurs 1 und Exkurs 2 folgt: Ethisches Handeln beim älteren Patienten in der Notaufnahme beginnt bereits damit,

seine Symptome ernst zu nehmen, das heißt

- auch bei atypischer Ausprägung richtig zu deuten und

- zielführend zu diagnostizieren sowie

- effektiv zu behandeln!

\section{Kasuistik 1: Der Patient hat Recht}

Ein 67-jähriger Patient aus einem Pflegeheim mit anamnestisch stattgehabtem Alkoholabusus und jetzt konsekutivem M. Korsakow wird von der Hausärztin telefonisch etwa so angekündigt: Er sei paranoid - er glaube, eine ernsthafte Erkrankung zu haben. Die Hausärztin sei in den letzten Wochen bereits zweimal die Woche bei inm gewesen und hätte ihn untersucht, aber keine Erkrankung feststellen können. Auch ein niedergelassener Psychiater habe den Patienten bereits mehrfach gesehen. Jetzt schickte die Hausärztin inn mit den Worten: Er wolle sich eine Krankenhauseinweisung „erschleichen“ - er habe gedroht, aus dem Fenster zu springen, wenn er nicht eingewiesen wird. Er sei jetzt hochgradig unruhig, agitiert und aggressiv. Die Hausärztin empfahl eine dringende psychiatrische Vorstellung bei Psychose mit Suizidalität. Die verständigten Kollegen der psychiatrischen Klinik insistierten auf einer somatischen Abklärung der evtl. deliranten Symptomatik, sodass zunächst die Vorstellung in unserer Notaufnahme erfolgte. Hier zeigte der Patient sich ruhig und gut führbar. Die kör- 
perliche Untersuchung und Auskultation ergab keine pathologischen Befunde bis auf eine Exsikkose, weil der Patient die letzten Tage im Pflegeheim aus Protest schon die Nahrung verweigert hatte. Laborchemisch zeigten sich eine Leukozytose und ein hohes CrP, sodass nach einen Infektfokus gefahndet wurde. Urinstatus, Röntgenthorax und Sonographie des Abdomens zeigten keinen Hinweis für einen Fokus, sodass zunächst bei „Delir bei Fieber unklarer Ursache" die Aufnahme erfolgen sollte. Als der Patient noch in der Notaufnahme eingekotet hatte, fiel der Pflegekraft (!) beim Wechseln des Inkontinenzmaterials eine ca. faustgroße druckdolente Schwellung perianal auf - ein Perianalabszess als Infektfokus. Der Patient zeigte SIRS-Kriterien, wurde den Chirurgen vorgestellt, zügig operiert und postoperativ wegen der Sepsis auf die Intensivstation übernommen. Tragischerweise war bei der Aufnahme des Patienten nicht bekannt geworden, dass er $2 \times 8 \mathrm{mg}$ Hydromorphon am Tag als Dauermedikation erhielt. Der Patient wurde postoperativ im Verlauf erneut delirant, was als „ITS-Koller" fehlgedeutet wurde, weil tragischerweise niemandem die Morphinmedikation bekannt war (die sich erst im Nachhinein herausstellte). Wegen des fortgesetzten Delirs und der anamnestisch beschriebenen Suizidalität erfolgte ein Konsil durch einen Psychiater, der dem Patienten Normalstationsfähigkeit bescheinigte. In der Nacht nach der Verlegung von der Intensivstation auf die Normalstation sprang der Patient - vermutlich im MorphinEntzugsdelir - aus dem Fenster und verstarb im Verlauf an den Folgen seiner Verletzungen.

Vier Dinge sind aus dieser Kasuistik ersichtlich:

- Auch ein „psychisch alterierter“ Patient kann recht haben.

- Der ältere Patient kann seine Beschwerden häufig nicht „präsentieren“, man muss nach innen „fahnden“.

- Ein Delir kann Ursache einer schweren Sepsis sein.

n Die Übermittlung der vollständigen Hausmedikation ist gerade bei multimorbiden und mit Polypragmasie behandelten Patienten mitunter lebenswichtig.

\subsection{Der alte Patient in der Notaufnahme am Lebensende: Therapieentscheidungen}

Viele ältere Patienten, die multimorbide sind und teilweise an chronischen oder malignen Erkrankungen mit infauster Prognose leiden (auch eine „ausgebrannte“ COPD oder eine fortgeschrittene Herzinsuffizienz sind von der Prognose her ähnlich zu betrachten wie eine Krebserkrankung), kommen im Endstadium ihrer Erkrankung in die Notaufnahme, weil entweder die Angehörigen (z.B. der ebenfalls betagte Ehepartner oder die weit weg wohnenden und berufstätigen Kinder) mit der Situation überfordert sind oder das Personal im Pflegeheim die Verantwortung für das Ableben des Patienten nicht übernehmen kann.

Oft tritt aber auch der Fall ein, dass Patienten mit akuten Erkrankungen in die Notaufnahme kommen, bei denen sich erst im Laufe der Untersuchung abzeichnet, dass die aktuelle Erkrankung möglicherweise das Ende des Lebens darstellt. Diese Szenarien stellen für jeden Notfallmediziner immer wieder eine Herausforderung dar, in der er sich meist allein mit der Therapiezielfindung konfrontiert sieht. Gründe sind fehlende Zeit, fehlende Informationen, fehlende Unterlagen oder nicht erreichbare beziehungsweise nicht entscheidungsfähige Angehörige (in der Schmitten et al. 2011).

Hier kommen unweigerlich zu medizinischen Fakten auch ethische Erwägungen mit in den Kreis der Überlegungen hinein. Trotz der mittlerweile in Deutschland gut 
etablierten Instrumente von Ethikberatung, wie Ethikkomitee, ethische Fallbesprechung und Ethikkonsil besteht das Problem, dass diese in der Situation der Erstbehandlung in der Notaufnahme oft nicht praktikabel sind (Trzeczak 2013). Gründe sind fehlende Zeit, fehlende Informationen, fehlende Unterlagen oder nicht erreichbare beziehungsweise nicht entscheidungsfähige Angehörige (in der Schmitten et al. 2011). Dies konnte in einer Umfrage unter Mitgliedern der Deutschen Gesellschaft für Interdisziplinäre Notfall- und Akutmedizin (DGINA) bestätigt werden (Padberg et al. 2014). Die Befragten signalisierten zum einen, dass in der Notaufnahme oft die Zeit fehlt, ein Ethikkomitee einzuberufen, dass sie sich aber andererseits mit der Entscheidung über Therapiemaximierung oder Therapiebegrenzung am Lebensende oft alleingelassen fühlen. Der Wunsch wurde laut nach schriftlichen Hinweisen, Handlungsempfehlungen oder einem „Gerüst“, an dem man sich im Notfall „entlanghangeln“ kann. Die AG „Ethik in der Notfall- und Akutmedizin“ der DGINA hat daraufhin eine „Checkliste Notfallpatienten“ entworfen, die auf der DGINA-Website abrufbar ist (http://www.dgina.de/pages/arbeitsgruppen/ethik.php). Andere, ähnliche Ansätze sind der Palliativnotfallbogen (Wiese et al. 2011) oder eine Mitteilung des Hausarztes an den Notarzt, die der Patient griffbereit hat (wie von in der Schmitten et al. 2011 vorgeschlagen). Vielversprechend sind auch Ansätze, die Patientenverfügung durch einen Code, der z.B. auf der Versicherungskarte des Patienten per Aufkleber vermerkt wird, jederzeit und überall online einsehbar zu machen (z.B. www. dipat.de).

Allgemeine Richtlinien und Handlungsempfehlungen, die zum Problem der Therapiezielfindung am Lebensende Stellung nehmen, finden sich in Tabelle 16.

Tab. 16 Wichtige Quellen zur Therapiezielfindung am Lebensende

\begin{tabular}{|l|ll|}
\hline Herausgeber & Titel & Quelle \\
\hline Bundesärztekammer & $\begin{array}{l}\text { Empfehlungen der Bundesärztekammer und } \\
\text { der Zentralen Ethikkommission bei der } \\
\text { Bundesärztekammer zum Umgang mit } \\
\text { Vorsorgevollmacht und Patientenverfügung } \\
\text { in der ärztlichen Praxis }\end{array}$ & $\begin{array}{l}\text { Deutsches Ärzteblatt Ig. 107/Heft } \\
\text { 18, 7. Mai 2010, S. 877-882 }\end{array}$ \\
\hline $\begin{array}{lll}\text { Bundesärztekammer } \\
\text { Grundsätze der Bundesärztekammer zur } \\
\text { ärztlichen Sterbebegleitung }\end{array}$ & $\begin{array}{l}\text { Deutsches Ärzteblatt lg. 108/ } \\
\text { Heft 7, 18. Februar 2011, S. 346-8 }\end{array}$ \\
\hline $\begin{array}{l}\text { Deutsche Interdiszipli- } \\
\text { näre Vereinigung für } \\
\text { Intensiv- und } \\
\text { Notfallmedizin (DIVI) }\end{array}$ & $\begin{array}{l}\text { Therapiezieländerung und Therapiebegren- } \\
\text { zung in der Intensivmedizin. Positionspapier } \\
\text { der Sektion Ethik der DIVI }\end{array}$ & www.divi-org.de \\
\hline $\begin{array}{l}\text { Deutsches Referenzzen- } \\
\text { trum für Ethik in den } \\
\text { Biowissenschaften } \\
\text { (DRZE) }\end{array}$ & $\begin{array}{l}\text { Verrel T., Simon A.: Patientenverfügungen. } \\
\text { DRZE-Sachstandsbericht }\end{array}$ & Freiburg i.Br., 2010 \\
\hline
\end{tabular}

Zum Problem der Ermittlung des Patientenwillens, der Einbeziehung von Patientenverfügung und Vorsorgevollmacht, zum tatsächlichen und mutmaßlichen Patientenwillen und zum „surrogate decision making“ unter Einbeziehung der Angehörigen sei auf die entsprechenden Kapitel im diesem Buch verwiesen. 
Patientenverfügungen stellen seit 2009 ein gesetzlich verankertes Instrument zur Ermittlung des mutmaßlichen Patientenwillens dar (Verrel u. Simon 2010). Die Schwierigkeit für den Notfallmediziner besteht darin, dass Patientenverfügungen trotz gesetzlicher Unterstützung immer noch nicht weit verbreitet und längst nicht immer vorhanden sind. Wenn vorhanden, sind sie oft in der Notfallsituation nicht schnell genug verfügbar und oft auch fraglich valide, das heißt die Verwertbarkeit für den Helfenden in der Notfallsituation, das Zutreffen auf die aktuelle Situation, ist nicht eindeutig sichtbar (in der Schmitten et al. 2011)

\section{Patientenverfügungen sind:}

nicht weit verbreitet,

- nicht immer vorhanden,

- wenn vorhanden, in der Notfallsituation oft nicht schnell genug verfügbar,

nenn verfügbar, ist die Validität, d.h. die Situationsbezogenheit fraglich (in der Schmitten et al. 2011).

Ein Problem mit Patientenverfügungen besteht auch darin, dass ihre Validität, d.h . die Gültigkeit in der entsprechenden Notfallsituation, oft nicht ohne Weiteres angenommen werden kann. So brachte einmal eine Angehörige eine Patientenverfügung ihres Ehemannes in die Notaufnahme, bei der es sich um einen der üblichen Vordrucke handelte. Der Patient hatte auf dem Vordruck alle vorhandenen Fragen mit „Ja“ beantwortet. Das führte zu so verwirrenden Angaben wie: „Ich wünsche die volle Ausschöpfung aller lebensverlängernden Maßnahmen: - Ja; Ich wünsche die Beendigung bzw. Nichtdurchführung lebensverlängernder Maßnahmen: - Ja; ...“. Darauf angesprochen, erwiderte die Ehefrau: „Ja, aber: Ich verstehe das Problem nicht, er hat doch alles ausgefüllt ...."Solche Antworten lassen einen an der Sinnhaftigkeit von Patientenverfügungen zweifeln. In der Schmitten (2010) schlägt diesbezüglich eine Beratung der Patienten vor Abfassung einer Patientenverfügung durch den Hausarzt vor - dies müsste jedoch, um im Praxisalltag durchführbar zu sein, von den Krankenkassen vergütet werden.

Die Änderung eines Patientenwillens trotz vorher abgefasster Patientenverfügung schildert Kasuistik 2.

\section{Kasuistik 2:}

Ein etwa 80-jähriger Patient wird mit einem akuten Koronarsyndrom kreislaufinstabil und kardial massiv dekompensiert in ein Krankenhaus der Grund- und Regelversorgung eingeliefert. In Anbetracht einer Patientenverfügung und in Absprache mit der Ehefrau wird auf eine Weiterverlegung des Patienten in ein Haus der Maximalversorgung zur Koronarangiographie verzichtet. Es wird konservative Therapie vereinbart, das heißt Herzinsuffizienzbehandlung, Sauerstoff und Schmerzbehandlung. In Anlehnung an die Patientenverfügung und in Absprache mit der Ehefrau soll auf Wiederbelebungsmaßnahmen verzichtet werden. Patient und Ehefrau sind über die Möglichkeit eines baldigen Ablebens informiert. Die Ehefrau sitzt am Bett des Patienten. Plötzlich kommt sie aufgeregt auf den Flur gelaufen und ruft laut: „Mein Mann braucht Hilfe!“. Der diensthabende Arzt folgt ihr ins Zimmer. Der Patient hat eine ventrikuläre Tachykardie, die im Verlauf in ein Kammerflimmern übergeht. Letztendlich 
gipfelt die Hilfe darin, dass der Patient defibrilliert wird. Dann erfolgt ein klärendes Gespräch mit der Ehefrau, in dem der Arzt das weitere Vorgehen besprechen möchte und auch noch einmal die Verlegung zur Koronarangiographie in ein Zentrum vorschlägt. (Die lebensgefährlichen Rhythmusstörungen wurden am ehesten durch eine Sauerstoffnot am Herzen durch eine Minderdurchblutung der Herzkranzgefäße hervorgerufen, insofern stellt hier die notfallmäßige Koronarangiographie eine sinnvolle Therapieoption dar). Die Ehefrau lehnt weiterhin ab und sagt, es solle nach dem Willen ihres Mannes nichts Lebensverlängerndes getan werden. Der Arzt entgegnet, dass sie, wenn sie diesen Willen zugrunde legen würden, zum jetzigen Zeitpunkt schon viel zu viel getan hätten. Ohne die Defibrillation wäre ihr Mann nicht mehr am Leben. Sie war es aber, die in der lebensbedrohlichen Situation auch nach Aufklärung um Hilfe gerufen und diese erwartet hat, die ja in dem Moment auch erfolgreich war. Schließlich willigt sie in die Verlegung ins nächste größere Krankenhaus zur Koronarangiographie ein. Nachdem der Mann zu sich gekommen war, wird das Vorgehen mit inm besprochen und er willigt ebenfalls ein. Zügig wird der Patient mit Notarztbegleitung verlegt (aus: Trzeczak 2010 in: Frewer et al. 2010).

Oft existieren auch Patientenverfügungen, die von Angehörigen zurückgehalten werden (Padberg et al. 2014). In diesem Fall, wenn Vorsorgebevollmächtigte oder Betreuer, statt einer Patientenverfügung Cültigkeit zu verschaffen, diese zurückhalten, müsste ein Betreuungsgericht zwischen Behandler und Angehörigen vermitteln, sobald zwischen diesen ein Dissens besteht (Verrel u. Simon 2010).

Allgemein kann zusammenfassend zu diesem Thema festgestellt werden, dass für die Durchführung einer Therapiemaßnahme folgende Voraussetzungen erfüllt sein müssen:

„Eine zulässige Behandlungsmaßnahme muss 2 Voraussetzungen erfüllen: 1) Für den Beginn oder die Fortführung der Behandlung besteht nach Einschätzung der behandelnden Ärzte eine medizinische Indikation. 2) Die Durchführung entspricht dem Patientenwillen." (Janssens et al. 2013)

Wichtig ist, noch einmal herauszuarbeiten, dass der Begriff der Indikation in zunächst die medizinische Indikation, die Aussagen über die medizinische Sinnhaftigkeit einer Behandlung trifft, und eine ärztliche Indikation unterteilt werden muss (Neitzke 2014). Diese wird dann mit dem Patienten oder den Angehörigen besprochen und der Patientenwille bzw. mutmaßliche Patientenwille bezüglich der Therapieoption ermittelt. Erst dann wird die eigentliche ärztliche Indikation zur Behandlung gestellt (Neitzke 2014). Das heißt, auch bei Vorliegen einer medizinischen Indikation kann bei Ablehnung der Behandlung durch Patienten und/oder Angehörige der Fall eintreten, dass eine ärztliche Indikation mangels Zustimmung nicht gestellt werden kann. Im Zweifel muss hier die Einwilligungsfähigkeit des Patienten, wenn nötig, psychiatrisch festgestellt werden bzw. bei Dissens zwischen Angehörigen/Betreuern und Behandler ein Betreuungsgericht eingeschaltet werden (Neitzke 2014).

Zur Stellung der medizinischen Indikation gehören laut DIVI folgende Überlegungen:

In einem ersten Schritt wird möglichst evidenzbasiert geprüft, ob die geplante Maßnahme prinzipiell geeignet ist, das angestrebte Therapieziel zu erreichen: Ist diese Krankheit mit der vorgesehenen Therapie erfolgreich zu behandeln? In einem zweiten Schritt wird geprüft, ob die geplante Maßnahme auch geeignet ist, dem individuellen Patienten in seiner konkreten Situation zu helfen: Profitiert dieser Patient mit dieser Erkrankung, ihrem Schweregrad, der Prognose und den vorliegenden Begleiterkrankungen von dieser Therapie?" (Janssens et al. 2013) 
Als nächstes folgt nach DIVI die Feststellung der Einwilligungsfähigkeit des Patienten. Hierbei werden verschiedene Grade der Einwilligungsfähigkeit unterschieden:

- der aktuell einwilligungsfähige Patient,

v der aktuell nicht einwilligungsfähige Patient mit (gültiger) Patientenverfügung,

v der aktuell nicht einwilligungsfähige Patient ohne (gültige) Patientenverfügung aber mit juristischem Stellvertreter (z.B. Betreuer oder Vorsorgebevollmächtigtem) und

v der aktuell nicht einwilligungsfähige Patient ohne (gültige) Patientenverfügung und ohne juristischen Stellvertreter (nach: Janssens et al. 2013)

Abschließend muss dazu bemerkt werden, dass eine nicht indizierte Maßnahme auch bei ausdrücklichem Patientenwunsch nicht durchgeführt werden sollte:

„Bei Patienten mit infauster Prognose kann nach den Grundsätzen der Bundesärztekammer zur ärztlichen Sterbebegleitung die Therapie begrenzt werden. Bei fehlender medizinischer Indikation dürfen Maßnahmen nicht durchgeführt werden, selbst dann nicht, wenn der Patient gegenteilige Wünsche hat." (Grübler 2011)

Diese Probleme kulminieren in der Situation, in der Wiederbelebungsmaßnahmen in der Notaufnahme durchgeführt oder unterlassen werden sollen (Mohr et al. 1997). Hierbei ist in kurzer Zeit eine Entscheidung zu treffen, der Arzt kennt in der Regel die Vorgeschichte des Patienten nicht und kann nicht oder nur eingeschränkt mit diesem kommunizieren (ebd.). Ein weiteres Problem sind fehlende schriftliche Vorausverfügungen und fehlende oder mit der Situation überforderte, nicht auskunftsfähige Angehörige (Trzeczak 2013; in der Schmitten 2011). In solch einer Situation besteht ein medizinisch-ethisches Dilemma (Trzeczak 2014): Über das Outcome des Patienten nach Reanimation gibt es bislang keine ausreichenden Daten (Lippert et al. 2010; Wegscheider 2012), und der Arzt, der mangels medizinischer Daten über den Patienten (Anamnese, Vorerkrankungen) Entscheidungshilfe in der Ethik sucht, sieht sich mit den heute allgemein akzeptierten vier Grundprinzipien der Medizinethik konfrontiert (Beauchamp u. Childress 2009): Fürsorge, Nicht-Schaden, Autonomie und Gerechtigkeit. Wie soll der Behandler nun aber über Fürsorge bzw. Patientennutzen und Nicht-Schaden befinden, wenn die Folgen seiner Behandlung (nämlich der Wiederbelebung) nicht bekannt sind?

Im Zweifelsfall ist hier der natürliche Wille des Patienten, d.h. der natürliche Lebenswille eines jeden Menschen, als bindend für den als „Geschäftsführer ohne Auftrag“ handelnden Arzt anzusehen (Lippert et al. 2010; Mohr et al. 1997). Dies wird auch in den Richtlinien der Bundesärztekammer zur Herz-Lungen-Wiederbelebung so angegeben.

Auf der Intensivstation kann in der Folge, wenn absehbar ist, dass eine Behandlung keinen therapeutischen Nutzen mehr bringt (z.B. Aufrechterhaltung biologischer Funktionen bei infauster Prognose ohne über längeren Zeitraum sichtbare Besserung des Zustandes), diese begrenzt oder unterlassen werden („allow natural death“). Dabei legt z.B. die österreichische Gesellschaft für Intensivmedizin fest:

"All grounds that justify withholding of therapy are also legitimate reasons for withdrawal of therapy" (Valentin 2008). (Alle Gründe, die ein Einfrieren der Therapie rechtfertigen, rechtfertigen auch die Beendigung dieser Therapie.) 
Als Gründe für Begrenzung oder Abbruch einer Therapie werden genannt (Valentin 2008):

- Progressive multi-organ failure under maximal intensive care medical therapy with no prospect of successful treatment of the cause (fortgeschrittenes Multiorganversagen unter maximaler Intensivtherapie ohne Aussicht auf erfolgreiche Behandlung der Ursache)

- Terminal failure of vital organs with no prospect of transplantation or adequate long-lasting organ substitution (terminales Versagen lebenswichtiger Organe ohne Aussicht auf Transplantation oder anderweitigen langfristigen Organersatz)

- Life-threatening intercurrent disease/complication or complete loss of autonomous vital functions after irreversible cerebral damage (lebensbedrohliche interkurrente Erkrankung/Komplikation oder kompletter Verlust autonomer Lebensfunktionen nach irreversibler Hirnschädigung)

- Terminal stage of chronic or malignant disease that no longer can be influenced by any therapy (Endstadium einer chronischen oder malignen Erkrankung, die nicht länger durch irgendeine Therapie beeinflusst werden kann)

Sollten Erkenntnisse über eine eventuelle Grunderkrankung des Patienten vorliegen, können folgende zwei Fragen bei der Entscheidungsfindung helfen (Trzeczak 2014):

1. Handelt es sich um eine Krankheit mit infauster Prognose und um eine palliative Situation? (s. Tab. 17)

2. Steht die aktuelle Akutsituation in Zusammenhang mit der Grunderkrankung? (s. Tab. 18).

Wenn eine palliative Grundsituation bei infauster Prognose vorliegt und die aktuelle Akutsituation nicht mit der infausten Grundkrankheit in Zusammenhang steht, kann als Entscheidungshilfe gelten, die aktuelle Akutsituation zu beherrschen und bezüglich der Grunderkrankung palliativ zu bleiben (Kasuistik 3). Sollte ein Zusammenhang zwischen der aktuellen Akutsituation und der palliativen Grunderkrankung bestehen (z.B. die aktuelle Akutsituation ein möglicher Endpunkt der Grunderkrankung sein) ist insgesamt Palliation geboten (Kasuistik 4).

Tab. 17 Frage nach der Grunderkrankung (nach Trzeczak 2014)

Patient mit chronischer Grunderkrankung und akuter Notfallsituation:

Grunderkrankung mit infauster Prognose?

Grunderkrankung mit erhaltener Therapieoption ohne infauste Prognose?

Frage nach dem ätiologischen/kausalen Zusammenhang Versuch der Beherrschung der akuten Notfallder akuten Notfallsituation mit der Grunderkrankung situation

Tab. 18 Frage nach dem Zusammenhang der Akutsituation mit der Grunderkrankung (nach Trzeczak 2014) Zusammenhang der Notfallsituation mit der Grunderkrankung?

\begin{tabular}{ll}
\hline la (Kasuistik 4) & Nein (Kasuistik 3) \\
\hline Palliation bezüglich Grunderkrankung & Versuch der Beherrschung der Notfallsituation \\
\hline Palliation bezüglich Notfallsituation & Nachfolgend palliatives Vorgehen bezüglich der Grunderkrankung \\
\hline
\end{tabular}




\section{Kasuistik 3}

Eine 73-jährige Patientin wurde mit Linksherzdekompensation bei supraventrikulärer Tachykardie (SVT) vorstellig. Zusätzlich besteht ein Karzinom mit unbekanntem Primärtumor („carcinoma of unknown primary“, CUP-Syndrom) mit hepatischer und lymphogener Metastasierung und linksseitigem malignem Pleuraerguss.

Nach Stabilisierung der Patientin durch nichtinvasive Beatmung und Frequenzkontrolle wurde sie auf die Normalstation verlegt. Über die Erkrankung und die infauste Prognose wurde mit der Patientin und den Familienangehörigen gesprochen. Dabei wurde bewusst auf erneute intensivmedizinische Maßnahmen verzichtet. Diese Entscheidung wurde von allen gemeinsam getragen.

Die Patientin wurde palliativ mit analgetischer und anxiolytischer Medikation behandelt und verstarb in der Folge an der fortschreitenden Grunderkrankung.

Die vorgestellte Patientin befand sich zusammengefasst am Endpunkt einer infausten Erkrankung (multipel metastasiertes fortgeschrittenes CUP-Syndrom). Eine Akutsituation, die primär nicht im Zusammenhang mit der Grunderkrankung steht (supraventrikuläre Tachykardie mit konsekutiver Linksherzdekompensation), kam akut komplizierend hinzu. Die Akutkomplikation wurde behandelt, bezüglich der Grunderkrankung wurde palliativ vorgegangen (aus: Trzeczak 2014).

\section{Kasuistik 4:}

Ein Patient mit metastasiertem Sigmakarzinom (beidseitige Lungen-, Leber- sowie ossäre Metastasen, peritonealen Adhäsionen) entwickelte eine akute oberer gastrointestinale Blutung bei Ösophagusvarizen, Grad II nach Savary und Miller.

Bei dem Patienten bestand ein ulzeriertes mittelgradig differenziertes tubulopapilläres Adenokarzinom im Sigma- und Colon-descendens-Bereich mit Infiltration aller Darmwandschichten und massivem Tumoreinbruch in das anhängende perimurale Fettgewebe. Die postoperative Tumorklassifikation war wie folgt: G2, pT3, pNo (0/17), pL0, pVo, pRo; initial: Stadium IIA nach Union internationale contre le cancer (UICC). In der Folge kam es 3 lahre später zur Detektion von Leber-und Lungenmetastasen sowie 4 lahre später zur zusätzlichen Diagnose einer ossären Metastasierung.

Bereits postoperativ war eine adjuvante Chemotherapie mit 5-Fluorouracil (5-FU) durchgeführt worden. Diese Therapie musste aufgrund einer schweren Mukositis zunächst pausiert werden und wurde dann niedriger dosiert fortgesetzt, bis sie schließlich abgebrochen werden musste. Nach Detektion der Metastasierung wurden mehrere Lungenmetastasenresektionen, eine Chemotherapie mit 4 Zyklen nach dem FOLFIRI-Schema, atypische Leberresektionen in Segment 2 und 3, insgesamt 15 Thermoablationen von Metastasen, 9 Zyklen einer Bevacizumabtherapie, 7 Zyklen einer Kombinationstherapie mit Bevacizumab und Irinotecan sowie eine Bisphosphonattherapie durchgeführt. Schließlich entschloss man sich zu einem begleitenden/beobachtenden Vorgehen. Aktuell wurden die Ösophagusvarizen mit insgesamt 5 Gummibandligaturen behandelt. In der begleitenden CT-Diagnostik zeigte sich ein deutlicher Progress der Erkrankung. Nach 4 Wochen wurde der Patient wieder aufgenommen, jetzt mit erneuter Allgemeinzustandsverschlechterung und erhöhten Cholestaseparametern. Daraufhin erfolgte eine endoskopisch retrograde Cholangiopankreatikographie (ERCP) mit Papillotomie und Stenteinlage in den linken und rechten Ductus hepaticus. Im Verlauf kam 
es nochmalig zu einer oberen gastrointestinalen Blutung, sodass wieder die Aufnahme auf die internistische Intensivstation erfolgte. Hier konnte nach erneuter Ligaturbehandlung und Transfusion von insgesamt 6 Erythrozytenkonzentraten zunächst eine Stabilisierung erreicht werden. Im Verlauf wurde jedoch deutlich, dass bei fortgeschrittener hepatischer Metastasierung mit konsekutiv nachlassender Gerinnung und steigendem portalem Hochdruck die Blutungsgefahr durch die Ösophagusvarizen eine Komplikation darstellte, die letztlich ihre Ursache in der fortschreitenden Grunderkrankung hatte.

Die Situation akzeptierend wurde nach Gespräch mit dem Patienten in Form einer „physicians order life sustaining treatment" (POLST) auf weitere intensivmedizinische Maßnahmen verzichtet und der Patient verstarb an den Folgen seines infausten Grundleidens. Schließlich musste eingesehen werden, dass die Beschwerden des Patienten im Verlauf Ausdruck seiner fortgeschrittenen Grunderkrankung waren und auftretende lebensbedrohliche Situationen letztlich einen möglichen Endpunkt dieser Erkrankung darstellten (aus: Trzeczak 2014).

Insgesamt kann zusammengefasst werden, dass zunächst die medizinische Indikation und dann der Patientenwille geprüft werden muss, bevor lebensverlängernde Maßnahmen bei alten Patienten am Lebensende durchgeführt werden. Die zitierten Richtlinien und Entscheidungshilfen können dabei eine Hilfe sein, wie die aufgeführten Kasuistiken zeigen. Sollte über einen Patienten gar keine Information vorliegen und auch kein Kontakt zu Angehörigen bestehen, ist im Zweifelsfall der Erhalt des Lebens als nächstliegende Therapieoption zu wählen - weiterführende Entscheidungen können und sollten dann im weiteren Verlauf getroffen werden.

\subsection{Abschluss: ethische Überlegungen zu Alter und Tod}

Nachdem aus gesundheitspolitischer, sozialer und medizinisch-praktischer Sicht auf die Problematik dieses Kapitels eingegangen wurde, sollen am Schluss einige Bemerkungen stehen, die das Ganze aus ethisch-philosophischer Sicht beleuchten sowie zum Nach- und Weiterdenken anregen sollen.

Es ist bereits erwähnt worden, dass zum gegenwärtigen Zeitpunkt die medizinischtechnischen Möglichkeiten zur Lebenserhaltung (soll heißen: zur Aufrechterhaltung biologischer Funktionen) nie so groß waren wie zuvor, dass aber auch nie zuvor in einer so breiten und offenen Diskussion diese Möglichkeiten derart in Frage gestellt wurden wie heute. Auch in Ärztekreisen wächst die Skepsis gegenüber einer Lebensverlängerung „um jeden Preis“ und es wird die Frage gestellt, ob es ein Ziel der Medizin sein kann, die menschliche Lebensspanne immer weiter zu verlängern. Hierzu wird ein breiter ethischer Diskurs gefordert (Barazzetti u. Reichlin 2011).

Das Alter wird allgemein als letzte Lebensphase nach Säuglingsstadium, Kindheit, Jugend und Erwachsenenalter angesehen (Helmchen et al. 2006). Die Definition ist schwierig, weil Alter als „Übergang von „Evolution zu Involution“, als „zunehmendes Überwiegen des Abbaus von Substanz, Struktur und Funktion gegenüber ihrem Aufbau und ihrer Entwicklung“, verschiedene Organsysteme zu unterschiedlichen Lebenszeiten betrifft und daher einen „heterochronen Prozess“ darstellt. Deshalb ist eine medizinisch-biologische Definition des Alters schwierig, und offiziell wird da- 
rum gegenwärtig das Ende beruflicher Tätigkeit als Beginn der Lebensphase des Alters angesehen (ebd.)

Hier nun sieht z.B. Maio bereits ein ethisches Problem: In einer Leistungsgesellschaft, in der Konsens darüber besteht, dass derjenige von der Gemeinschaft anerkannt wird, der auch etwas zu ihrer Erhaltung beiträgt, sind die Alten, die nicht mehr aktiv am Berufsleben und damit zur Vermehrung des Bruttosozialproduktes beitragen, von dieser Anerkennung ausgenommen (Maio 2014). Der ältere Mensch wird von der Wirtschaft und der Politik heute als „Konsument“ gesehen, viele Branchen bieten „altersgerechte“ Produkte an: altersgerechte Wohnungen, Seniorenreisen, seniorengerechte Autos, Treppenlifte usw. In der Werbung wird der Senior als aktiver, selbständiger Konsument dargestellt. Das setzt viele ältere Leute unter Druck, diesem Bild des „aktiven Seniors“entsprechen zu müssen. Maio hält dagegen, dass gerade die Situation der Angewiesenheit aufeinander - sei es aus Gründen der Pflegebedürftigkeit, der Demenz oder schlicht der Einsamkeit nach Versterben eines Ehepartners - zur conditio humana des alten Menschen gehört und uns als menschlicher Gesellschaft spiegelt, dass wir alle soziale Wesen (,zoon politikon“, d.h. „politisches Tier“; Aristoteles - d.A.) sind (Maio 2014).

Die Realität sieht aber leider so aus, dass viele ältere Menschen ihr Leben als „nicht mehr lebenswert" betrachten, wenn sie unter unheilbaren progressiven Krankheiten leiden und somit ihre Selbständigkeit verlieren. Dies führt zur öffentlichen Diskussion über Sterbehilfe, Euthanasie und Beihilfe zum Suizid, die in verschiedenen europäischen Ländern unterschiedlich geregelt werden (Helmchen et al. 2006).

Demgegenüber steht ein interessantes Phänomen aus der Pharma-Industrie: Viele Medikamente werden danach beurteilt und ihre Wirksamkeit daraufhin getestet, ob sie lebensverlängernd wirken. Die Lebensqualität wird dabei nicht in Betracht gezogen. Der Terminus der „quality adjusted life years“ (QALY) findet derzeit lediglich bei medizinökonomischen Überlegungen Platz (u.a.: Weinstein et al. 2009). Das heißt, die älteren Menschen bekommen einerseits Medikamente, die ihr Leben verlängern, andererseits weiß die Gesellschaft nicht, was sie mit diesen älteren Menschen „anfangen soll“" (wenn wir die Überlegungen von Maio 2014 und Helmchen 2006 hinzuziehen).

Wir sollten das Altwerden mit allen seinen Begleiterscheinungen als eine „conditio humana“ akzeptieren und die Voraussetzungen dafür schaffen, dass hier eine gesellschaftliche Akzeptanz einsetzt.

Das nächste Problem, was hier erörtert werden soll, ist das Lebensende und der Umgang mit Sterben und Tod.

Bereits 1987 konstatierte Hans Jonas ein „Recht zu sterben“(Jonas 1987). Er begründete dies aus dem Recht auf Leben: Das Leben ist etwas Naturgegebenes, wir selbst haben schließlich keinen Einfluss darauf, ob wir geboren werden oder nicht. Da wir dieses Naturgegebene uns selbst zugestehen (und damit auch jedem anderen menschlichen Wesen um uns herum zugestehen müssen), gehört es zu den sogenannten „unveräußerlichen Rechten“ (ebd.). Jonas führt weiter aus, dass der Tod zum Leben dazugehört und somit jeder auch im Laufe seines Lebens die Gelegenheit haben soll, sich mit dem Tod als „Vollendung des Lebens“ auseinanderzusetzen. Maio spricht von dem „Bogen des Lebens“, der „voll werde“ (Maio 2014). Für Heidegger ist der Tod die Vollendung des Lebens und die Bedingung des „Ganz-Sein-Könnens“, wobei die 
Existenz des Daseins mit dem Eintreten dieses „Ganz-Seins“ aber eben auch erlischt. Er spricht darum von einem „Sein zum Tode“ (Heidegger 1993; Trzeczak 2010) - „nascentes morimur“, es sei an den Beginn dieses Kapitels erinnert! Ähnlich formuliert es auch Rieger (2013): Er benutzt die Metapher vom „dem Tod entgegenwachsen“, vom Sterben nicht als „Abbruch“, sondern als „Vollendung“(ebd.).

Den Tod als zum Leben dazugehörig zu begreifen, bedeutet jedoch nicht die Notwendigkeit für den Einzelnen, sein Leben bis zum Eintreten des Todes unter Kontrolle zu halten („mein Tod gehört mir“).

Das würde bedeuten, dass jeder, der, aus welchen Gründen auch immer, sein Leben aktuell als „nicht mehr lebenswert“ erachtet oder glaubt, seine Autonomie (d.h. seine „Kontrolle“ über die Dinge) zu verlieren, den Freitod wählen darf. Diese Auffassung ist es, die z.B. der aktuellen Diskussion über Tötung auf Verlangen und Beihilfe zum Suizid immer wieder neuen Zündstoff verleiht. Maio argumentiert dazu folgendermaßen: Genauso wenig, wie wir eine Wahl haben, ob wir ins Leben treten möchten oder nicht, haben wir eine Wahl, ob, wann und wie wir aus dem Leben scheiden möchten.

„Wer im Zusammenhang des Verbots der aktiven Sterbehilfe von Zwang zu Leben spricht, geht implizit davon aus, dass das Leben nicht etwas Gegebenes ist, sondern eine Option, ja das Resultat einer persönlichen Entscheidung." (Maio 2014)

Nährboden für solche Ansichten ist wiederum der befürchtete „Verlust von Autonomie“, wobei hier Autonomie falsch verstanden wird als das - angenommene - NichtAngewiesensein einer Person auf andere. Hier kann man wiederum mit Maio argumentieren, dass es gerade ein Merkmal des menschlichen Lebens ist, dass wir permanent auf andere angewiesen sind und diese auf uns: Eltern, Geschwister, Partner, Kinder, Kollegen, der Bäcker um die Ecke, Nahrungsmittel- und Kleidungshersteller ... Maio bezeichnet Angewiesensein als eine „Grundsignatur des ganzen Lebens“ und sagt, dass sie „nicht das Ende der Autonomie ist, sondern eine Crundbedingung, überhaupt zu sein, und somit eine Voraussetzung für Autonomie“ (Maio 2014).

Wünschenswert wäre es, in dieser Angewiesenheit die Würde des Angewiesenen zu respektieren bzw. seine persönliche Autonomie zu erhalten. Das Alter ist nach Maio „eine Erinnerung daran, was der Mensch ist: ein endliches Wesen, das nicht alleine existieren kann“.

Sollte jedoch ein Patient - gleich, ob im irreversiblen Koma oder bei Bewusstsein unter einer unheilbaren fortschreitenden Erkrankung leiden (auch eine terminale Herzinsuffizienz oder eine „ausgebrannte“ chronisch-obstruktive Bronchitis können von der Prognose her ähnlich infaust sein wie eine Krebserkrankung), wirft dieses Problem nach Jonas (1987) „jenseits dieses Raumes der „Rechte“ die Frage nach der letzthinnigen Aufgabe der ärztlichen Kunst auf ...“, die da lautet: „Gehört das bloße, hinauszögernde Zurückhalten vor der Todesschwelle zu den echten Zielen oder Pflichten der Medizin?“, oder wie es Barrazzetti et al. (2011) formulierten: „Life extension a biomedical goal?“ Jonas meint dazu, dass „selbst eine transzendentale Pflicht zu leben auf seiten des Patienten keine Nötigung zu leben von seiten des Arztes rechtfertigt“. „Das neuartige Phänomen von Patientenohnmacht gekoppelt mit der Macht todesverzögernder Techniken unter öffentlicher Obhut“ verlangt hier nach Jonas nach einer „Verteidigung des Rechtes zu sterben“ (dessen Begründung bei Jonas weiter oben bereits dargestellt wurde) durch eine Besinnung auf die „wirkliche Berufung 
der Medizin“. Jonas plädiert hier für z.B. eine Schmerzlinderung, die durch ihre Dosierung eine Lebens- und damit Leidensverkürzung in Kauf nimmt. Dies stellt heute z.B. in der Palliativmedizin eine gängige Praxis dar. ${ }^{9}$

\section{Fazit für die Praxis:}

- Ältere Patienten werden in Zukunft zunehmend die Notaufnahmen frequentieren. Dies liegt zum einen am demographischen Wandel und zum anderen an der Zunahme der Multimorbidität.

- Ältere Patienten benötigen mehr Zeit bei Anamnese und Untersuchung. Der Kontakt zum Rettungs- und Pflegepersonal sowie zum Arzt ersetzt häufig fehlende Sozialkontakte.

- Ältere Patienten bieten häufig wenige oder atypische Symptome, man muss nach ihren Erkrankungen "fahnden“. Das zieht einen hohen Aufwand an Diagnostik nach sich, wodurch sich der Aufenthalt dieser Patienten in der Notaufnahme verlängert.

- Therapieentscheidungen in Akutsituationen bei älteren Patienten sollten die Grund- und Begleiterkrankungen und deren Prognose sowie Patientenverfügungen bzw. Betreuer mit einbeziehen. Nur eine therapeutische Maßnahme, die medizinisch indiziert ist und dem (aktuell erfragten oder vorausverfügten/ mutmaßlichen) Willen des Patienten entspricht, sollte auch durchgeführt werden. Ist eine Maßnahme nicht indiziert, sollte sie auch bei Wunsch des Patienten/der Angehörigen nicht durchgeführt werden (bei Dissens zwischen Betreuern/Angehörigen und dem Behandler ist das Betreuungsgericht einzuschalten).

- Abhängigkeit und Pflegebedürftigkeit muss keinen Verlust an Autonomie bedeuten. Erst in der Abhängigkeit von anderen entwickelt sich unser Leben von der Geburt bis zum Tod, sie schafft erst die Voraussetzung für Autonomie. Lassen wir unsere Patienten spüren, dass wir sie trotz Abhängigkeit und Pflegebedürftigkeit mit Respekt behandeln und ihre Autonomie und Würde respektieren.

- Lebensverlängerung soll nicht Leidensverlängerung bedeuten. Sollte eine schwere Erkrankung mit hohem Leidensdruck für den Patienten und die Angehörigen bei infauster Prognose bestehen, ist eine Leidensverminderung auch mit Inkaufnahme einer Lebenszeitverkürzung - die ja dann doch nur eine Verkürzung des vorliegenden Leidenszustandes bedeuten würde - ethisch geboten.

9 Vor diesem Hintergrund erscheint es unverständlich, dass kürzlich ein österreichischer Anästhesist verklagt wurde, weil er einer chronisch schwerkranken Patientin zur Leidensminderung Morphium verabreicht hat - die Morphiumdosis soll nach Ansicht des Gerichts „den Tod herbeigeführt haben“ (http://www.salzburg.com/nachrichten/salzburg/chronik/sn/artikel/patientin-starbdroht-salzburger-arzt-mordanklage-168657/).

Es gibt dazu bereits eine Stellungnahme der österreichischen Gesellschaft für Ethik und Recht in der Notfall- und Katastrophenmedizin ÖGERN: http://www.oegern.at/keine-kriminalisierung-der-palliativmedizin-oegern-publiziert-stellungnahme 


\section{Literatur}

Bäcker G, Naegele G, Bispinck R, Hofemann K, Neubauer I (2010) Sozialpolitik und soziale Lage in Deutschland. Band 2: Gesundheit, Familie, Alter und Soziale Dienste. Verlag für Sozialwissenschaften, Wiesbaden

Barazzetti G, Reichlin M (2011) Life extension: a biomedical goal? Scientific prospects, ethical concerns. Swiss Med Weekly 141: w13181

Beauchamp TL, Childress JF (2009) Principles of Biomedical Ethics. Oxford University Press, New York

Bürger M (1960) Altern und Krankheit als Problem der Biomorphose. Georg Thieme Verlag, Leipzig

Bundesärztekammer (2010) Empfehlungen der Bundesärztekammer und der Zentralen Ethikkommission bei der Bundesärztekammer zum Umgang mit Vorsorgevollmacht und Patientenverfügung in der ärztlichen Praxis. Dtsch Ärztebl 107: 877-882

Bundesärztekammer (2011) Grundsätze der Bundesärztekammer zur ärztlichen Sterbebegleitung. Dtsch Ärztebl 108: 346-8

Cernic K, Likar R, Pinter G (2013) Zentrale Notfallaufnahme und zentrale Notaufnahme für ältere Menschen. In: Pinter G, Likar R, Schippinger W, Janig H, Kada O, Cernic K (Hrsg.) Geriatrische Notfallversorgung. Strategien und Konzepte. Springer Verlag, Wien

Grönemeyer D (2003) Mensch bleiben. High-Tech und Herz - eine liebevolle Medizin ist keine Utopie. Herder Verlag, Freiburg-Basel-Wien

Grübler B (2011) Wann soll das Leben zu Ende gehen? Therapiebegrenzung bei infauster Prognose. Dtsch Ärztebl 108(26): 1473-1476

Heidegger M (1993) Sein und Zeit. Max-Niemeyer-Verlag, Tübingen

Helmchen H, Kanowski S, Lauter H (2006) Ethik in der Altersmedizin. (Grundriss Gerontologie, Band 22). Kohlhammer Verlag, Stuttgart

Heppner HJ, Wiesner R, Schuster S, Thiem U, Christ M, Singler K (2014) Bedeutung der demographischen Entwicklung für die Notfallmedizin. Notfall Rettungsmed 17: 494-499

Hien P, Neubart R, Pilgrim RR (2013) Moderne Geriatrie und Akutmedizin. Springer-Verlag, Berlin/Heidelberg

in der Schmitten I, Rixen S, Marckmann G (2011) Patientenverfügungen im Rettungsdienst (Teil 1). Geklärte und offene Fragen nach Verabschiedung des Patientenverfügungsgesetzes. Notfall Rettungsmed 14: 448-58

Janssens U, Burchardi H, Duttge G, Erchinger R, Gretenkort P, Mohr M, Nauck F, Rothärmel S, Salomon F, Schmucker P, Simon A, Stopfkuchen H, Valentin A, Weiler N, Neitzke G (2013) Therapiezieländerung und Therapiebegrenzung in der Intensivmedizin. Positionspapier der Sektion Ethik der Deutschen Interdisziplinären Vereinigung für Intensiv- und Notfallmedizin. Med Klin Intensivmed Notfallmed 108: 47-52

Jonas H (1987) Technik, Medizin und Ethik. Praxis des Prinzips Verantwortung. Insel Verlag, Frankfurt am Main

Kettler D (1997) Ethik in der Notfallmedizin: Eine Einführung. In: Mohr M, Kettler D (Hrsg.) Ethik in der Notfallmedizin. Präklinische Herz-Lungen-Wiederbelebung. Springer-Verlag, Berlin/Heidelberg

Klotz I (2013) Steigende Lebenserwartung: Länger gesund oder krank? In: Pinter G, Likar R, Schippinger W, Janig $\mathrm{H}$, Kada 0, Cernic K (Hrsg.) Geriatrische Notfallversorgung. Strategien und Konzepte. Springer Verlag, Wien

Köller M (2014) Geriatrische Aspekte bei betagten Notfallpatienten. Notfall Rettungsmed 17: 484-487

Krug H (2009) Der alte Patient: Herausforderungen an die ethische wie fachliche Kompetenz des Arztes. Ethik Med 21: 101-111

Latham LP, Ackroyd-Stolarz S (2014) Emergency Department Utilization by Older Adults: a Descriptive Study. Canadian Geriatrics Journal 17 (4): 118-125

Lippert FK, Raffay V, Georgiou M, Steen PA, Bossaert L (2010) Ethik der Reanimation und Entscheidungen am Lebensende. Sektion 10 der Leitlinien zur Reanimation 2010 des European Resuscitation Council. Notfall Rettungsmed 13: 737-44

Lowthian JA, Smith C, Stoelwinder JU, Smith DV, MC Neil J), Cameron PA (2013) Why older patients of lower clinical urgency choose to attend the emergency department. Intern Med I 43 (1): 59-65

Maio G (2014) Medizin ohne Maß? Vom Diktat des Machbaren zu einer Ethik der Besonnenheit. TRIAS Verlag, Stuttgart

Neitzke G (2014) Indikation: fachliche und ethische Basis ärztlichen Handelns. Med Klin Intensivmed Notfallmed 109: 8-12 
Padberg I, Esser A, Lomberg L, Trzeczak S (2014) Ethische Probleme im Umgang mit Reanimation und Patientenverfügung in der Notaufnahme. Ergebnisse einer Onlineumfrage unter den Mitgliedern der DGINA. Notfall Rettungsmed 17: 500-506

Rieger M (2013) Dem Tod entgegenwachsen. Sterben als Abbruch oder Vollendung? In: Bieneck A, Hagedorn H-B, Koll W (Hrsg.) An den Grenzen des Lebens. Theologische, medizinethische und spirituelle Zugänge. Reihe Neukirchener Theologie. Neukirchener Verlagsgesellschaft, Neukirchen-Vluyn

Schippinger W, Langsenlehner U, Muchar H (2013) Somatische Veränderungen im Alter. In: Pinter G, Likar R, Schippinger W, Janig H, Kada 0, Cernic K (Hrsg.) Geriatrische Notfallversorgung. Strategien und Konzepte. Springer Verlag, Wien

Schreiber W (2014) Der „alte Notfallpatient“. Notfall Rettungsmed 17: 483

Terborg C (2012) Septische Enzephalopathie. Med Klein Intensivmed Notfallmed 107: 629-633

Trzeczak S (2010) Hoffnung und Verantwortung am Lebensende. Heideggers Existenzialontologie in der klinischen Praxis. In: Frewer A, Bruns F, Rascher W (Hrsg.) Hoffnung und Verantwortung - Herausforderungen für die Medizin. Jahrbuch Ethik in der Klinik 3, Verlag Königshausen \& Neumann, Würzburg

Trzeczak S (2013) Ethische Kompetenz und praktische Erfahrung. Dtsch Arztebl 110: 706-707

Trzeczak S (2014) Das medizinisch-ethische Dilemma von Reanimationsentscheidungen bei Notfallpatienten. Notfall Rettungsmed 17: 613-619

Trzeczak S (2015) Der Palliativpatient als Notfallpatient. Med Klin Intensivmed Notfallmed 110(4): 278-286

Tschugg H (2012) Der alte Mensch in der Intensivmedizin. In: Salomon F (Hrsg.) Praxisbuch Ethik in der Intensivmedizin. Medizinisch Wissenschaftliche Verlagsgesellschaft, Berlin

Valentin A, Druml W, Steltzer H, Wiedermann Cl (2008) Recommendations on therapy limitation and therapy discontinuation in intensive care units: Consensus Paper of the Austrian Associations of Intensive Care Medicine. Intensive Care Med. 34: 771-776

Verrel T, Simon A (2010) Patientenverfügungen. Rechtliche und ethische Aspekte. Ethik in den Biowissenschaften - Sachstandsberichte des DRZE 11. Verlag Karl Alber Freiburg/München

Wegscheider K (2012) Gibt es eine Evidenz für medizinische Aussichtslosigkeit? Notfall Rettungsmed 15: 667-670 Weinstein MC, Torrance G, McGuire A (2009) QALYs: The Basics. Value In Health 12: S5-S9

Wiese CHR, Duttge G, Weber AK, Zausig YA, Ruppert D, Hanekop GG, Graf BM (2009a) Notfallmedizinische Betreuung von Palliativpatienten am Lebensende. Juristische Beurteilung notfallmedizinischer Handlungsweisen - retrospektive Fallbetrachtung zur medizinischen Indikation und zum Patientenwillen. Anaesthesist 58: 1097-1106

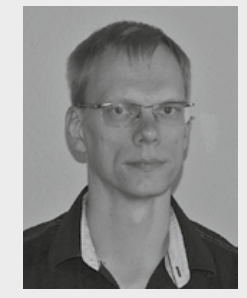

\section{Dr. med. Stefan Trzeczak, M.A.}

Studium der Humanmedizin in Rostock 1992-1998, begleitend Gasthörer am Institut für Philosophie. Facharzt für Chirurgie 2006, Facharzt für Innere Medizin 2012, Zusatzbezeichnung Notfallmedizin 2010. Berufsbegleitend Absolvierung des Masterstudiengangs „Philosophie im Europäischen Kontext“ an der FernUniversität Hagen (Abschluss Master of Arts M.A. 2010). Oberarzt in der Zentralen Notaufnahme des HELIOS Hanseklinikums Stralsund. Gründer und Sprecher der AG „Ethik in der Notfall- und Akutmedizin“ der Deutschen Gesellschaft interdisziplinäre Notfall- und Akutmedizin (DGINA) und Mitglied der Akademie für Ethik in der Medizin (AEM). Veröffentlichung von Vorträgen, Artikeln und Buchbeiträgen zum Thema Ethik in der Medizin, insbesondere ethische Probleme bei Reanimationen und Entscheidungsfindung am Lebensende. 